\title{
Claude-François Baudez (1932-2013)
}

\section{Aurore Monod Becquelin et Pierre Becquelin}

\section{OpenEdition}

\section{Journals}

Édition électronique

URL : https://journals.openedition.org/jsa/13780

DOI : 10.4000/jsa. 13780

ISSN : $1957-7842$

\section{Éditeur}

Société des américanistes

\section{Édition imprimée}

Date de publication : 17 septembre 2014

Pagination : 259-269

ISSN : 0037-9174

\section{Référence électronique}

Aurore Monod Becquelin et Pierre Becquelin, « Claude-François Baudez (1932-2013)», Journal de la

Société des américanistes [En ligne], 100-1 | 2014, mis en ligne le 17 octobre 2014, consulté le 02

septembre 2022. URL : http://journals.openedition.org/jsa/13780 ; DOI : https://doi.org/10.4000/jsa. 13780 


\section{NÉCROLOGIES}

\section{Claude-François BAUDEZ (1932-2013)}

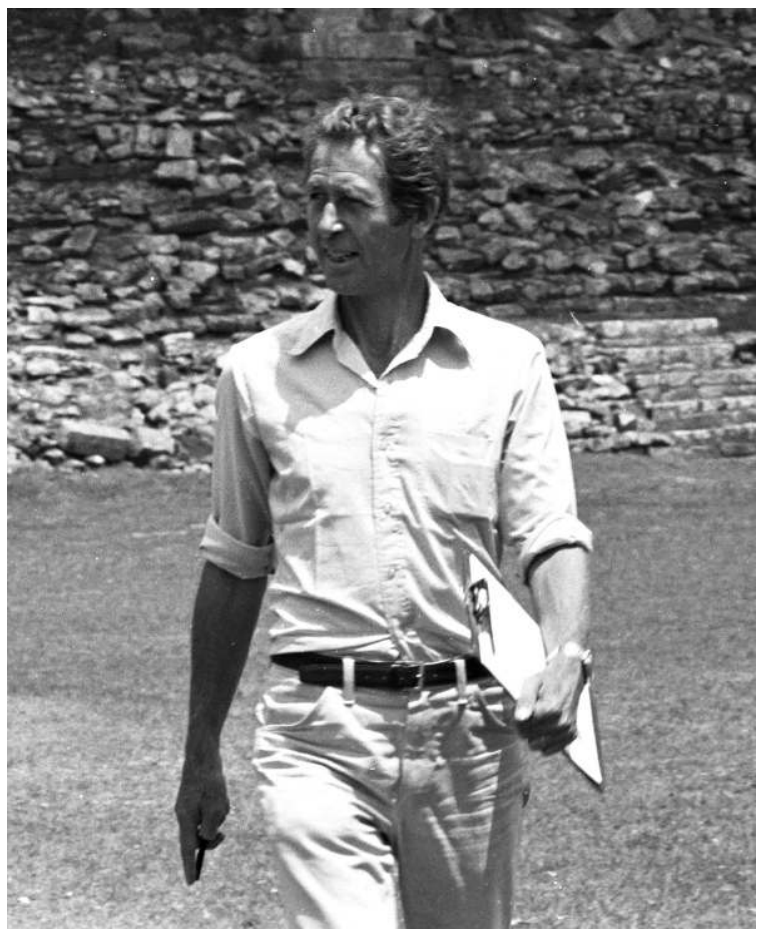

Claude-François Baudez, directeur du Proyecto arqueólogico Copán (1977-1980). Cliché Proyecto arqueólogico Copán (droits réservés).

Claude-François Baudez est né à Paris en 1932 et il s'y est éteint le 13 juillet 2013. Il est reconnu par la communauté scientifique internationale comme un archéologue et iconologue mésoaméricaniste remarquable.

À l'Institut d'ethnologie de l'université de Paris il obtient, en 1956, les certificats d'ethnologie et de linguistique après celui de sociologie, tout cela pendant les longues années du service militaire de l'époque. C'est à ce moment-là qu'il suit les séminaires de Guy Stresser-Péan et Claude Lévi-Strauss. Son premier terrain, en 1957, s'effectue au Costa Rica. Il complètera sa licence avec un 
certificat de littératures d'Amérique latine en 1960 et il entre la même année au CNRS comme attaché de recherche. En 1964, il soutient sa thèse de troisième cycle «Recherches archéologiques dans la vallée du Tempisque, Guanacaste, Costa Rica » laquelle sera publiée en 1967. Toute sa carrière s'est déroulée au CNRS, où il était devenu directeur de recherche. Il a bénéficié de l'appui constant du ministère français des Affaires étrangères (commission des fouilles) et de plusieurs institutions internationales pour mener à bien ses nombreux travaux à l'étranger : Costa Rica, Honduras, Mexique.

Ses recherches en Amérique centrale, à partir de ses premiers terrains au Costa Rica, entre 1957 et 1960, puis au Honduras, dans la deuxième moitié des années 1960, furent consacrées fondamentalement à l'établissement de séquences culturelles, lesquelles étaient à l'époque encore fort imprécises. Point d'orgue de ce premier ensemble de travaux, il choisit d'effectuer des fouilles sur le grand site hondurien de Los Naranjos, un établissement protégé par des fossés et qui livra une séquence chronologique allant du Préclassique au Postclassique (Baudez et Becquelin 1973). Dans l'élaboration de cette séquence, l'étude qu'il réalisa de la céramique allia avec succès approche typologique et approche modale. Ces diverses recherches lui permirent de rédiger une synthèse de l'archéologie de l'Amérique centrale, publiée en 1970 à Genève dans la collection « Archaeologia Mundi », qui reste encore une référence. Il y montre en particulier clairement la division que l'on peut établir entre zone influencée par les civilisations mésoaméricaines et zone liée aux cultures sud-américaines. Dans la suite de sa carrière il n'oubliera jamais complètement l'Amérique centrale de ses débuts, son dernier travail sur la région, concernant le Diquis et réalisé en collaboration avec Borgnino, Laligant et Lauthelin, ayant été publié en 1993.

À partir des années 1970, Claude se tourna résolument vers la civilisation maya. Le site de Toniná est le déclencheur qui marque le tournant de sa vocation, et ce, non seulement sur le plan géoculturel mais aussi sur celui des thématiques. En effet, en plus des fouilles qu'il effectuait à cet endroit, et à l'occasion de l'étude des sculptures du site, il se découvre alors un intérêt, qui devint immédiatement une passion, pour les réalisations de l'art maya et pour les sens à leur donner. De nombreuses publications témoignent de ce tournant dans sa carrière, à commencer par les trois premiers tomes de Tonina, une cité maya du Chiapas (Mexique), parus respectivement en 1979 et 1982. Les travaux de la mission française au Chiapas dirigés par Pierre Becquelin et qui se poursuivirent jusqu'en 1980 le conduisirent donc à l'étude précise de l'iconographie de cette cité et à l'établissement d'un vocabulaire descriptif modèle, ce dernier mis au point après de nombreux échanges avec plusieurs collègues, notamment Ian Graham et Peter Mathews. Toutefois, lorsqu'il publie en 1984, avec Pierre Becquelin, Les Mayas dans la prestigieuse collection conçue par André Malraux, «L'univers des formes ", il hésite encore à introduire ses nouvelles interprétations iconologiques que l'on trouvera plus tard, au fil de plusieurs articles et, sous une 
forme synthétique dans Une Histoire de la religion des Mayas. Du panthéisme au panthéon (2002 pour l'édition française, 2004 pour la version espagnole). L'image de la religion maya qu'il présente dans cet ouvrage est aussi originale parce qu'il adopte résolument, à son sujet, une perspective historique, ce qui implique de scruter les changements dans les cultes et les croyances et de donner autant d'importance aux crises et aux ruptures qu'aux permanences. C'est en fait un livre qu'il faut relire, car les pistes d'interprétation qu'il offre sont beaucoup plus subtiles que ce que bien des spécialistes y ont vu : il permet en effet d'échapper à la confrontation stérile entre continuité et rupture.

La passion de Claude pour l'iconographie et les relations de l'image à la société maya se confirme complètement lorsque, à la suite de Gordon Willey, il prend la direction du programme international de recherche à Copán au Honduras entre 1977 et 1980. S'il supervise alors l'ensemble des recherches, prospections dans la vallée et fouilles du site et de ses alentours comprises, rapidement c'est à la sculpture du site qu'il s'intéresse personnellement, et il continuera à le faire dans les années suivantes. À l'époque, bien d'autres chercheurs s'intéressaient à l'iconographie et aux inscriptions de Copán, mais de façon bien plus ponctuelle que Claude qui, lui, développait une analyse systématique et méthodique de tout le corpus de la sculpture. Coordinateur des trois volumes de Introducción a la arqueología de Copán, il publie aussi, et surtout, en 1994, aux presses de l'université d'Oklahoma, Maya sculpture of Copán: the iconography, un ouvrage qui demeure la meilleure introduction à l'iconologie du site.

D'un point de vue méthodologique, l'art maya n'est, à ses yeux, pas séparable de la religion, des formes de pouvoir et des croyances. Dès le début de son travail de terrain, dans une lettre à Michel Izard de 1959, citée par Olivier Herrenschmidt, il écrit qu'on assigne à l'archéologie un rôle historique en négligeant ses contributions à l'anthropologie. Comprendre ce que signifie le fait de représenter l'oblige, pour atteindre une véritable iconologie, à associer un sens à chaque graphisme de la manière la plus indiscutable possible, et cela exige un travail de comparaison iconographique considérable. La méticulosité avec laquelle il intériorise chaque détail des images - par l'œil, en observant, et par la main, en dessinant - coïncide avec un questionnement sur le savoir qui se manifeste dans ces éléments mais ordonne aussi les compositions plus larges. Car Baudez joint à la minutie dans l'observation une vision élargie des sites et de la position de ses objets d'étude dans leurs contextes, les ensembles architecturaux notamment, à Copán en particulier, plus tard à Palenque.

Sur un domaine qui concerne l'esthétique et l'art autant que l'archéologie, et parce qu'il était au contact d'ethnologues, d'ethnolinguistes, de linguistes et d'épigraphistes, il est remarquable que les questions que Claude-François Baudez se posaient aient été, dès l'abord, d'ordre anthropologique : la royauté, les captifs, le sacré, l'espace rituel, les attributs de la religion, le sacrifice. La guerre et ses 
apparats surgissent dès son analyse des sculptures de Tonina. Le thème de la royauté sacrée s'impose aussi à lui très vite comme un domaine d'exploration, mais à étudier dans une perspective comparative : sa nature, ses attributs, ses pouvoirs. L'autosacrifice prend enfin de plus en plus d'importance dans ses interrogations, ce qui le pousse à aborder un problème philosophique et logique autant que mayaniste et mésoaméricaniste : comment, par une chaîne d'assimilations, le sacrifice de l'autre devient le substitut des multiples formes du sacrifice de soi. Il interroge à titre comparatif les Olmèques, les Teotihuacans et surtout les Aztèques. Les résultats de ces questionnements font l'objet de ses dernières publications, en particulier La douleur rédemptrice : l'autosacrifice précolombien paru en 2012, puis en traduction espagnole en 2014 au Mexique.

À l'occasion des séminaires et conférences qu'il donne, il provoque de nombreuses discussions de la part de ses collègues : archéologues, mais aussi experts d'autres disciplines, américanistes ou spécialistes d'autres continents, dont les organisations sociales et symboliques peuvent être sources de comparaisons. Soucieux d'élargir l'audience autour du cercle des mayanistes ou des mésoaméricanistes, il ne néglige pas la publication d'ouvrages de synthèse depuis celui, déjà mentionné, de 1970 sur l'Amérique centrale jusqu'à Les Mayas, en 2004, aux éditions Les Belles Lettres. Dans le même esprit, et tournés vers un public élargi, on peut citer son étude du peintre Waldeck, parue en 1993 chez Hazan, et le petit ouvrage, élaboré avec Sydney Picasso, Les cités perdues des Mayas, dans la collection «Découvertes » de Gallimard qui, traduit dans de nombreuses langues, lui valut des dizaines de milliers de lecteurs.

Le très grand nombre de contributions qu'on doit à Claude-François Baudez, seul ou en collaboration, montre la richesse de son travail de diffusion des connaissances. Mais il joignit aussi à cette tâche une présence assidue et des interventions répétées dans les établissements et institutions où son enseignement pouvait toucher à la fois un public non averti et des savants, des étudiants aussi bien que des collègues, et cela à l'étranger comme en France.

De fait, l'un des points de son activité qui permet de comprendre l'importance de sa participation au débat scientifique, donne matière aussi à parler de lui de façon plus intime : il s'agit de la vie collective qu'il n'a cessé de mener avec ses collègues en France, sa participation aux divers groupes de travail auxquels il s'associait avec persévérance.

Assidu au séminaire de l'EPHE « Religions de l'Amérique précolombienne », organisé par Michel Graulich, qu'il a même remplacé en 1996-1997, ce dont il se disait très heureux, il a accompagné avec constance ce dernier, surtout à partir des années 2000 lorsqu'il s'est attaché à l'analyse du sacrifice humain. Ensuite, entre 2007 et 2012, il y a donné pas moins de seize conférences : dans ce séminaire, repris alors par Danièle Dehouve, les problématiques abordées marquent la progression de sa pensée en même temps que la continuité de ses préoccupations. 
Parmi les nombreux thèmes traités de façon comparative, on retiendra, par exemple, l'étude des dépôts rituels découverts dans le Templo Mayor de la ville de Mexico et publiés par Leonardo López Luján (1993), où il s’intéresse notamment à leur disposition en strates verticales, plusieurs agencements constituant une recréation du monde. En mettant en parallèle ces dépôts avec d'autres découverts en zone maya (dans les remplissages au-dessus des sépultures par exemple), Baudez propose l'existence d'une pratique généralisée en Mésoamérique de dépôts cosmogoniques enterrés.

Un autre thème qu'il approfondira est celui de l'égalité des sexes. Après plusieurs auteurs, il compare la fête Tlacaxipehualiztli, "écorchement des hommes ", à une autre fête mensuelle, Ochpaniztli, " usage des balais ». Il remarque que, si la première apparaît comme masculine, Ochpaniztli met surtout en scène des femmes et que les deux, vues ensemble, partagent l'année en deux moitiés équivalentes, l'une masculine, l'autre féminine. Il va d'ailleurs plus loin encore dans l'analyse iconologique : dans une conférence intitulée « Le troisième sexe ", il compare les fêtes Ochpaniztli et Tititl représentées dans le Codex de Florence : il montre que les rituels, parce qu'ils traitent des femmes à deux moments de leur vie (âge mûr et vieillesse), fondent la subdivision de la société en groupes constitutifs et représentent donc des opérateurs classificatoires.

Bien évidemment, royauté sacrée et autosacrifice sont les domaines de ses contributions principales, et le travail sur la royauté maya classique est central dans son œuvre. En particulier à propos du roi de Copán longtemps nommé « 18-Lapin », Claude souligne la dimension cosmique du roi, comparé au soleil sortant de terre lors de son intronisation. Son corps est un microcosme et les actes rituels d'autosacrifice qu'il pratique promeuvent la fertilité. Une des particularités de son approche, élaborée à partir des années 1980, fut de partir de l'image du captif conçu comme l'opposé structural du roi et une image en creux de celui-ci (voir, par exemple, les emblèmes de la captivité versus ceux de la royauté), chaque complexe étant délimité temporellement, spatialement et par contraste. Chemin faisant, il reconsidère le thème des rituels sanglants et propose de repenser ensemble les activités de guerre, de jeux et de batailles rituelles, ce qui lui permet d'élaborer une nouvelle typologie à deux catégories seulement : la guerre, d'un côté, les batailles rituelles, de l'autre (auxquelles appartiennent les jeux de balle), dont le but est de faire des morts et des blessés par l'utilisation du hasard, c'est-à-dire du sort.

L'iconographie du sacrifice restera jusqu'en 2013 son centre d'intérêt privilégié. Il amplifie les démonstrations de Graulich selon lequel le sacrifice de soi est un rite créateur et réparateur, ainsi qu'un moyen de pression sur les dieux. Il s'agit d'un rite d'une grande souplesse capable de s'adapter aux occasions les plus diverses ; une des phases d'un cycle sans fin de dons et de contre-dons, d'humiliation et de rédemption, de souffrance et de bien-être. 
C'est en participant aux réunions, ateliers et colloques du Groupe d'enseignement et de recherche maya (GERM) que Claude a bâti et raffiné sa vision de l'autosacrifice. En se reportant à cette période, on prend la mesure de la dynamique de sa réflexion.

Le GERM était né dans les années 1970-1980 de l'obstination de Pierre Becquelin et de l'équipe de Toniná, avec Claude, bien sûr, et plusieurs jeunes collègues et étudiants, pour engager des réflexions collectives et interdisciplinaires sur le monde maya. Sollicités également pour donner des cours d'archéologie au département d'ethnologie de l'université de Nanterre, associé au Laboratoire d'ethnologie et de sociologie comparative fondé par Éric de Dampierre, les chercheurs du groupe inaugurèrent une longue suite d'enseignements de l'archéologie mésoaméricaniste à Paris X. C'est dans ce cadre que débuta l'engagement infaillible de Claude dans les activités du GERM, soumettant toutes ses avancées en iconologie au groupe de travail qui avait été constitué. En 2000, grâce à l'installation dans un bâtiment commun, la Maison de l'archéologie et de l'ethnologie René Ginouvès, le GERM resserra les liens qui existaient déjà entre les deux principaux laboratoires porteurs (LESC et ARCHAM auquel Claude Baudez appartenait) en attirant aussi plusieurs membres d'autres laboratoires et départements. Là plus qu'ailleurs, Claude put tester de façon comparative ses recherches, en particulier sur les similarités et dissemblances avec le monde aztèque, mais également avec d'autres parties du continent américain, de l'Amazone aux Andes. Derrière pierres et d'objets, auxquels il cherchait un sens, ou images, dont il sondait la signification, il fouillait la religion et le pouvoir. $\mathrm{Au}$ gré des thèmes choisis et des spécialistes extérieurs invités, Claude, qui était toujours présent, s'offrait à la discussion : ainsi, la question « politique et religion dans la civilisation classique maya » fut confrontée à la vision d'une sinisante spécialiste des royaumes combattants, les rapports entre archéologie et religion chez les Mayas discutés par un indianiste ou un ethnohistorien du monde aztèque. Le sujet sur les figures de la royauté alimenta de nombreux mois de discussion tant avec des péruanistes et des amazonistes que des indianistes ou des africanistes.

Lorsque Claude Baudez débuta sa carrière d'archéologue, devinait-il que sa curiosité, sa passion de la comparaison et un infatigable intérêt pour les autres disciplines allaient le conduire à utiliser des outils opératoires propres à la linguistique, à tirer profit de notions fines issues de la topologie ? Au hasard de ses articles dans les ouvrages collectifs du GERM, on peut aisément illustrer sa manière de faire de la recherche et son style.

En 2003, dans Espacios mayas, il offre un article intitulé « Las aguas terrestres entre los antiguos mayas: representaciones y rituales ». Dans les lignes qui suivent, on perçoit à la fois sa prudente rigueur, qui lui interdit toute extrapolation, et, en même temps, sa précision dans les descriptions qui font surgir, par la justesse des termes les plus concrets, un réalisme poétique de bon aloi : 
On ne sait pas bien ce que vont faire les danseurs et les musiciens, où, quand, comment et pourquoi [...], mais ce que l'on peut dire c'est que grâce à la musique, la danse et les gestes, on va donner ou redonner de la vie et de l'énergie, réactiver un milieu aquatique qui symbolise la vie, le renouveau, l'abondance. Au son des trompettes, au rythme des hochets, des tambours et des carapaces de tortue, les nénuphars mordillés par les poissons frémissent, les écrevisses entourent le seigneur des eaux - que nous appelons le monstre aquatique -, les crabes agitent leurs pinces, les crocodiles font claquer leurs mâchoires et les canards nasillent. C'est ainsi que l'on comprend mieux la fonction de la structure 12 de Copan qui donne aux acteurs d'un rituel semblable l'espace nécessaire à leurs évolutions. [traduction des auteurs A. M. B. et P. B.]

Au cours du travail dans un des ateliers de l'ACI Géographie du sacré du GERM, Claude peaufine un essai sur le parallélisme dans les images mayas de la période classique, qu'il publiera en 2010 dans l'ouvrage qui fit suite au colloque international conclusif de ces ateliers. C'est dans la partie linguistique intitulée « Parallélisme » et non dans la section archéologique, que se situe son article « $L a$ tierra y sus máscaras », texte dans lequel il scrute les variantes iconographiques de ce procédé linguistique et cognitif.

No es sólo por ser agricultores que los mesoamericanos prestan tanta importancia a la tierra; es también por sus cualidades y funciones a la vez diversas y contradictorias, por su carácter de refugio misterioso de las fuerzas de la vida como de las de la muerte, y por ser sitio de notables transformaciones.[...] A esta multiplicidad de funciones corresponde una multiplicidad de formas que recurren a varias figuras estilísticas para ilustrarlas (2010, p. 195)

La signification des figures en iconologie rapproche les analyses d'un phénomène syntaxique et sémantique, le diphracisme : à une unité sémantique ou significative ("structure d'une image ») correspondent deux ou plusieurs termes (exemple du monstre terrestre) ; les « associations »-attributs, figures, ensembles, emboîtages, etc. - montrent une certaine fixité en ce qui concerne le sens global, mais des variantes dans la composition de ce sens « pour révéler des oppositions ou des nuances ». Les effets sémantiques de ces différentes configurations relationnelles portent sur une grande variété de thèmes : opposition ou complémentarité de nature des éléments, cyclicité saisonnière, ambivalence des entités.

[El mascarón] es usado tanto para marcar oposiciones y diferencias como para expresar la permanencia gracias a la repetición de una forma inmutable. Se manifiesta a veces como sujeto, participando activamente en una composición; a veces como atributo para atribuir o confirmar el carácter terrestre a un animal emblemático, una alegoría, un ser mítico, y hasta a un edificio. (ibid., p. 205)

Dans ce même recueil et dans le travail qui lui sert d'épilogue « El paralelismo de las páginas 33 y 34 del Códice Borgia », sa méthodologie apparaît limpide et 
rigoureuse à la fois. Il inclut le contexte comme élément majeur de l'analyse de manière à rendre compte de subtiles variations, une des difficultés de l'iconologie maya. La première phrase est significative de sa façon de penser :

Investigaciones recientes me llevaron a comparar dos grandes celebraciones aztecas [...] [la que] tenía como propósito [...] asimilar a los guerreros mexicas con sus víctimas [...] [y la que] buscaba asimilar a las mujeres con los guerreros. (2010, p. 539)

Et plus loin on lit :

Sólo es comparable lo que es a la vez semejante y diferente. La analogía observable entre dos íconos, que radica en la forma, el color, la estructura ylo el contexto, incita a compararlos para poner de relieve la diferencia que tiene sentido. Imágenes de la misma estructura, en particular, provocan la comparación que tiene el efecto de evidenciar equivalencias, variantes, oposiciones y hasta variaciones. (2010, p. 550)

C'est ainsi qu'il dénombre les oppositions ponctuelles, les contrastes d'ensemble dans une même structure, les constantes - co-texte du parallélisme -, les exclusives, et qu'il donne une interprétation neuve aux deux pages de ce codex. Il y trouve un « exemple notable de parallélisme en iconographie », et démontre ce qu'il appelle l'équilibre des sexes dans une cosmologie, idée reprise de ses conférences précédentes, mais définie ainsi dans la mesure où elle n'est ni cosmogonie, ni narration de rituels, puisqu'il prend en compte ce qu'implique la notion de genre linguistique et qu'il l'utilise à bon escient en iconologie.

En 2012, Claude publie un article brillant, un de ses tout derniers, dans un recueil collectif en ligne qui fit suite à un travail de deux ans du GERM autour du modèle topologique de la frontière épaisse. Le thème abordé l'occupait déjà depuis un certain temps.

Chez trois ethnies qui pratiquaient la guerre de capture (Aztèques, Iroquois et Tupinamba), le rituel avait pour fonction d'assimiler le prisonnier à son vainqueur, la victime au sacrifiant, l'Autre à Soi. Les guerres auraient en commun les épisodes suivants : deux phases d'agressivité, l'une suivant la capture et l'autre précédant l'exécution, sacrifice, mort et renaissance, prohibitions alimentaires et deuil. Ces épisodes créent une fusion entre le meurtrier et sa victime. La réflexion de Claude Baudez sur la nature structurellement équivalente du sacrificateur et du sacrifié est un prolongement des analyses menées dans son livre La douleur rédemptrice. L'autosacrifice précolombien, qui fait du sacrifice mésoaméricain un épiphénomène de l'autosacrifice. Mais pour être en syntonie avec l'enjeu de la recherche collective qui était menée, ce sont les étapes de cette transformation qui sont analysées comme les moments successifs du franchissement d'une frontière épaisse : il étudie donc les successions d'actes, d'objets ou de discours qui font de l'ennemi un parent, transforment l'hostilité en impuissance et assimilent l'Autre à Soi. En scrutant l'apparition et la présence de microdétails dans les descriptions, il précise une chronologie de l'assimilation qui ne suit, ni celle d'une narration, ni 
celle de la description de scènes manifestes. Il nuance le dualisme amérindien en montrant le passage d'une catégorie à une autre qui lui est ontologiquement opposée, argumentant que le guerrier et le captif aztèques sont des êtres de frontière et que chacun passe de son statut à celui de l'autre par un ensemble parfaitement ordonné d'une multitude d'actes, de paroles et d'objets. Claude suit là magistralement les mouvements du double jeu de mort et de vie. Il éclaire la nature de la frontière ontologique qui est franchie : vie et mort sont une même réalité pour les Amérindiens, la vraie frontière se situe entre le soi et l'autre et c'est là qu'intervient le rituel majeur, pour faire de ce passage, non une métaphore, mais de véritables expériences de vie puisqu'elles touchent à la pratique : parenté, relations politiques, expressions normées des émotions, parties du corps, nourritures, choix d'une couleur, d'un ornement, d'une plume. Cet article, condensé, impeccable dans la démonstration, est un grand exemple de la description raisonnée du passage des frontières.

Le GERM, qu'il a animé pendant plus de quarante ans avec générosité, ne peut que garder en mémoire la qualité de sa participation à la recherche. Inoubliables demeurent son allant, ses rires et son étonnement combatif lorsqu'on lui proposait d'intervenir sur un thème trop improvisé ou une idée inutile. De Claude Baudez et du travail effectué collectivement avec lui, on retiendra sa fidélité à trois des principes qui nous tenaient et nous tiennent à cœur : la confrontation et, si possible, la collaboration entre disciplines, la comparaison avec d'autres cultures et contextes, en particulier amazonien et océanien, le partage des connaissances et l'enseignement. On ne saurait enfin oublier de mentionner sa fidélité à bien d'autres institutions et lieux de recherche en France (CNRS, EPHE, EHESS, INALCO) et ses contributions à la principale revue française du domaine, le Journal de la Société des américanistes. Comment ne pas terminer cette évocation de ce qu'il fut sans se référer à cette ambivalence constitutive de sa personne, entre doute et passion, que très tôt il énonçait lui-même dans des lettres à son ami Olivier Herrenschmidt.

Pour revenir à ma profession, je m'étonne de ne pas l'exécrer encore : chaque fois que je l'exerce, c'est dans le doute, l'ignorance, l'angoisse [...] (novembre 1959)

J'aime tant mon métier, car il me permet de me dépenser physiquement [...] et en même temps me permet de satisfaire mes quelques prétentions intellectuelles (avril 1961).

Son ardeur à la discussion et son inventivité restent précieuses pour ses collègues et ses amis et demeurent un modèle de ce que peuvent être des relations idéales de travail et de gaieté.

Aurore Monod Becquelin et Pierre Becquelin 


\section{RÉFÉRENCES CITÉES}

BAUDEZ Claude-François

1967 Recherches archéologiques dans la Vallée du Tempisque, Guanacaste, Costa Rica, Centre National de la Recherche Scientifique/IHEAL, coll. « Travaux et Mémoires »18, Paris.

1970 Amérique Centrale, Nagel, coll. « Archaeologia Mundi », Genève.

1993 Jean-Frédéric Waldeck, peintre: le premier explorateur des ruines mayas, Hazan, Paris.

1994 Maya sculpture of Copán : the iconography, University of Oklahoma Press, Norman/Londres.

2002 Une histoire de la religion des Mayas, Albin Michel, coll. «Bibliothèque Histoire », Paris.

2003 «Las aguas terrestres entre los antiguos mayas: representaciones y rituales », in Alain Breton, Aurore Monod Becquelin et Mario Humberto Ruz (éd.), Espacios mayas: representaciones, usos, creencias, Universidad Nacional Autónoma de México/Centro de estudios mexicanos y centroamericanos, coll. " Estudios de cultura maya », Mexico, pp. 463-488.

$2004 \quad$ Una historia de la religión de los antiguos mayas, UNAM/CEMCA/Centre culturel et de coopération pour l'Amérique centrale, coll. " Arqueología », Mexico.

2004 Les Mayas, Les Belles Lettres, coll. « Guide Belles Lettres des Civilisations », Paris.

2010 «La tierra y sus máscaras », in Aurore Monod Becquelin, Alain Breton et Mario Humberto Ruz (éd.), Figuras mayas de la diversidad, UNAM, Mérida, pp. 195-206.

2010 «El Paralelismo de las páginas 33 y 34 del Códice Borgia » in Aurore Monod Becquelin, Alain Breton et Mario Humberto Ruz (éd.), Figuras mayas de la diversidad, UNAM, Mérida, pp. 539-566.

2012 La douleur rédemptrice : l'autosacrifice précolombien, Riveneuve éditions, Paris.

2012 «Le passage de la frontière », Ateliers d'anthropologie, 37 [En ligne : http://ateliers.revues.org/9198].

BAUDEZ Claude-François (éd.)

1983 Introducción a la arqueología de Copán, Honduras, 3 vol., Instituto Hondureño de Antropología e Historia, Tegucigalpa.

Baudez Claude-François et Pierre BeCQuelin

1973 Archéologie de Los Naranjos, Honduras, Mission archéologique et ethnologique française au Mexique, coll. « Études mésoaméricaines »2, Mexico.

1984 Les Mayas, Gallimard, coll. « L’Univers des formes », Paris.

Baudez Claude-François, Nathalie Borgnino, Sophie LaLigant et Valérie Lauthelin

1993 Investigaciones arqueológicas en el delta del Diquís, CEMCA/DRCSTE Mexico/San José de Costa Rica. 
Baudez Claude-François et Sydney Picasso

1987 Les cités perdues des Mayas, Gallimard, coll. « Découvertes », Paris.

BECQuelin Pierre et Claude-François BAUDEZ

1979 Tonina, une cité maya du Chiapas, Tome I, Mission archéologique et ethnologique française au Mexique, coll. "Études mésoaméricaines» 6-1/Éditions Recherche sur les civilisations, Paris/Mexico.

1982 Tonina, une cité maya du Chiapas, Tomes II, III, Mission archéologique et ethnologique française au Mexique, coll. "Études mésoaméricaines » 6-2/Éditions Recherche sur les civilisations, Paris/Mexico.

LóPEZ LUJÁN Leonardo

1993 Las ofrendas del Templo Mayor de Tenochtitlan, INAH, Mexico.

Pour une bibliographie complète de Claude-François Baudez, se reporter à :

http://gemeso.com/membres/claude-baudez/ 